\title{
H- and He-burning Central Stars and the Evolution to White Dwarfs
}

\author{
T. Blöcker \\ Max-Planck-Institut für Radioastronomie, 53121 Bonn, Germany
}

\begin{abstract}
The structure and evolution of central stars of planetary nebulae (CSPNe) is reviewed. CSPNe represent the rapid transitional stage between the Asymptotic Giant Branch (AGB) and the white-dwarf domain. It is shown that the whole evolution off the AGB through the central-star regime depends on the evolutionary history. The detailed evolution into a white dwarf is controlled by the internal stellar structure which, in turn, is determined by the duration of the preceding AGB evolution and therefore by the AGB mass-loss history. The evolution of hydrogen-deficient central stars has been a matter of debate since many years. Convective overshoot appears to be a key ingredient to model these objects. Various thermal-pulse scenarios with inclusion of overshoot are discussed, leading to surface abundances in general agreement with those observed for Wolf-Rayet central stars.
\end{abstract}

\section{Introduction}

Central stars of planetary nebulae (CSPNe) represent the rapid transitional stage between the Asymptotic Giant Branch (AGB) and the white-dwarf domain. Since the pioneering work of Paczyński (1971), CSPNe have continued to be in the focus of evolutionary calculations. For instance, Schönberner (1979, 1983) demonstrated how the detailed evolution into a central star depends on the previous AGB evolution, i.e. on the thermal-pulse cycle at the tip of the AGB and the shut down of the heavy AGB mass loss. The dependence of the CSPN evolution on the thermal-pulse cycle was investigated in full detail later by Iben (1984), and existing model grid calculations were complemented by the computations of Wood \& Faulkner (1986). The next stage of calculations included the consideration of appropriate initial-final mass combinations based on empirical and semi-empirical AGB mass-loss prescriptions (Vassiliadis \& Wood 1993, 1994; Blöcker 1995a,b). Further reviews on the AGB and post-AGB evolution and comparisons of the above calculations are given, e.g., by Iben (1995), Habing (1996), Wood (1997) and Schönberner (1997).

While the evolution of hydrogen-rich central stars appears to be adequately understood, the formation of hydrogen-deficient central stars has been a matter of debate for many years. Convective overshoot turned out to be a prerequisite to model the abundances of objects as the Wolf-Rayet central stars (Herwig et al. 1999, Blöcker 2001, Herwig 2001a). 


\section{Moving off the AGB}

Stars evolving along the AGB suffer from increasingly strong stellar winds with mass-loss rates up to $\sim 10^{-4} \mathrm{M}_{\odot} / \mathrm{yr}$ (Habing 1996), eroding up to $\sim 80 \%$ of the initial mass and terminating the AGB evolution when the envelope mass has dropped to $\approx 10^{-2} \mathrm{M}_{\odot}$ (Schönberner 1979). The stars move off the AGB, become central stars of planetary nebulae, and finally reach after exhaustion of nuclear burning the stage of white dwarfs. The complete evolution of a $3 \mathrm{M}_{\odot}$ main-sequence star into a $0.625 \mathrm{M}_{\odot}$ white dwarf is illustrated in Fig. 1.

One the upper AGB, the He burning shell becomes recurrently unstable, giving rise to the so-called thermal pulses (Schwarzschild \& Härm 1965, Weigert 1966), during which the luminosity of the He shell increases rapidly for a short time of $100 \mathrm{yr}$ to $10^{6} \ldots 10^{8} \mathrm{~L}_{\odot}$. The huge amount of energy produced in the $\mathrm{He}$ shell forces the development of a pulse-driven convection zone which mixes products of He burning, i.e. carbon and oxygen, into the intershell region. Because the hydrogen shell is pushed concomitantly into cooler domains, hydrogen burning ceases temporarily, allowing the envelope convection to proceed downwards after the pulse, to penetrate those intershell regions formerly enriched with carbon (and oxygen), and to mix this material to the surface ( $3^{\text {rd }}$ dredge up). After the pulse, $\mathrm{H}$ burning re-ignites and provides again the main source of energy. Accordingly, the evolution off and beyond the AGB depends on the thermalpulse cycle phase $\phi$ (fraction of the time span between two subsequent pulses), with which the stars move off the AGB. Fig. 2 shows the surface luminosity and the contributions of the $\mathrm{H}$ and $\mathrm{He}$ burning shell as a function of $\phi$ for the tenth pulse cycle of a $3 \mathrm{M}_{\odot}$ AGB sequence. The post-AGB evolution is dominated by He burning for $0 \leq \phi \leq 0.15$. For $0.15 \leq \phi \leq 0.3$ both nuclear shell sources contribute similar luminosity fractions, for $0.3 \leq \phi \leq 1.0 \mathrm{H}$ burning determines the nuclear energy production (Iben 1984). If the thermal-pulse cycle-phase is sufficiently large $(\phi>0.75)$, a last thermal pulse can occur during the post-AGB evolution transforming a $\mathrm{H}$ burning into a He burning model. The flash forces the star to expand rapidly to Red Giant dimensions, and the remnant quickly evolves back to the AGB ("born again scenario"). There, it starts its post-AGB evolution again, but now as a He burning object (Iben 1984).

The details of the transformation of AGB stars into white dwarfs is crucially determined by the treatment of mass loss on the AGB and beyond. Observations indicate that mass-loss should decrease by orders of magnitudes during the transition to the central-star regime (Perinotto 1989). However, at which temperature this strong decrease takes place is only barely known. The transition time scale $t_{\mathrm{H}}$ of a $\mathrm{H}$-burning model is determined by the shrinkage of its envelope mass $\Delta M_{\mathrm{e}}$ due to burning and mass loss, divided by the burning rate $\dot{M}_{\mathrm{H}}$ and the mass-loss rate $\dot{M}_{\mathrm{w}}$, i.e. $t_{\mathrm{H}}=\Delta M_{\mathrm{e}} /\left(\dot{M}_{\mathrm{H}}+\dot{M}_{\mathrm{w}}\right.$ ) (Schönberner 1983). On the upper AGB, the evolution is controlled by mass loss whose rates are orders of magnitudes larger than those of burning $\left(\dot{M}_{H} \sim 10^{-7} \mathrm{M}_{\odot} / \mathrm{yr}\right.$ for $M_{\mathrm{H}}=0.6 \mathrm{M}_{\odot}$ ). After shut down of the heavy AGB mass loss, burning takes over and mass loss is only of minor importance. The earlier mass loss decreases, the longer are the transition times, and the larger is the probability to obtain a final thermal pulse and thus a He burning model. In the calculations of Vassiliadis \& Wood (1994), the strong AGB mass-loss was switched off close to the AGB $\left(T_{\text {eff }}<5000 \mathrm{~K}\right)$ and the earlier the more massive the remnants are, leading to 

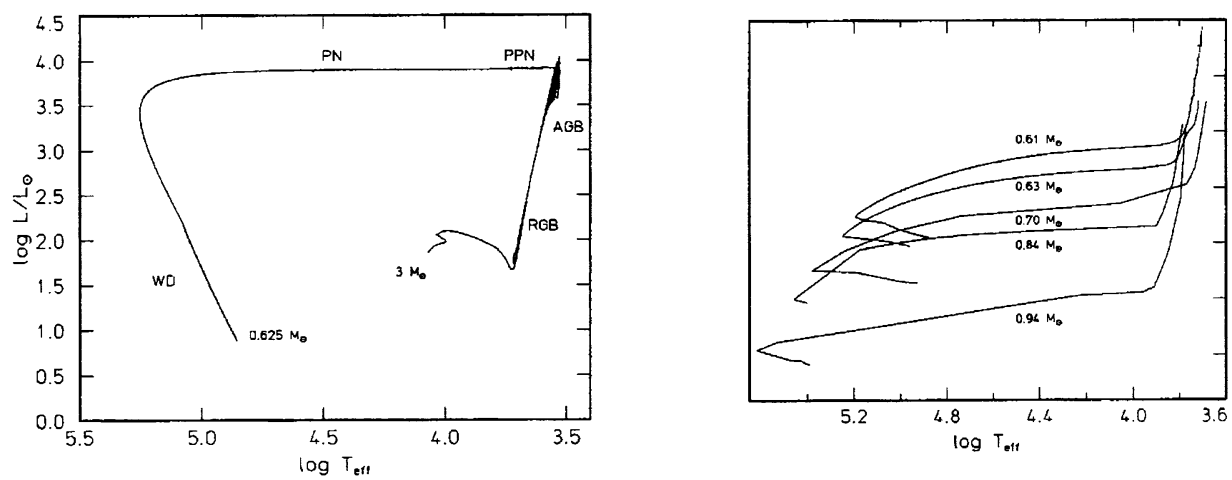

Figure 1. Left: Evolutionary path of a star with an initial mass of $3 \mathrm{M}_{\odot}$ from the main sequence through the AGB towards the whitedwarf stage. On the AGB, the star suffers from 21 thermal pulses until mass loss terminates the AGB evolution. The final mass is $0.625 \mathrm{M}_{\odot}$. During the post-AGB evolution the model is burning hydrogen until extinction of the shell source. Right: Envelope mass vs. effective temperature for hydrogen burning post-AGB models (Blöcker 1995b). The initial and remnant masses are $\left(3 \mathrm{M}_{\odot}, 0.61 \mathrm{M}_{\odot}\right),\left(3 \mathrm{M}_{\odot}, 0.63 \mathrm{M}_{\odot}\right)$, $\left(4 \mathrm{M}_{\odot}, 0.70 \mathrm{M}_{\odot}\right),\left(5 \mathrm{M}_{\odot}, 0.84 \mathrm{M}_{\odot}\right)$, and $\left(7 \mathrm{M}_{\odot}, 0.94 \mathrm{M}_{\odot}\right)$, resp.

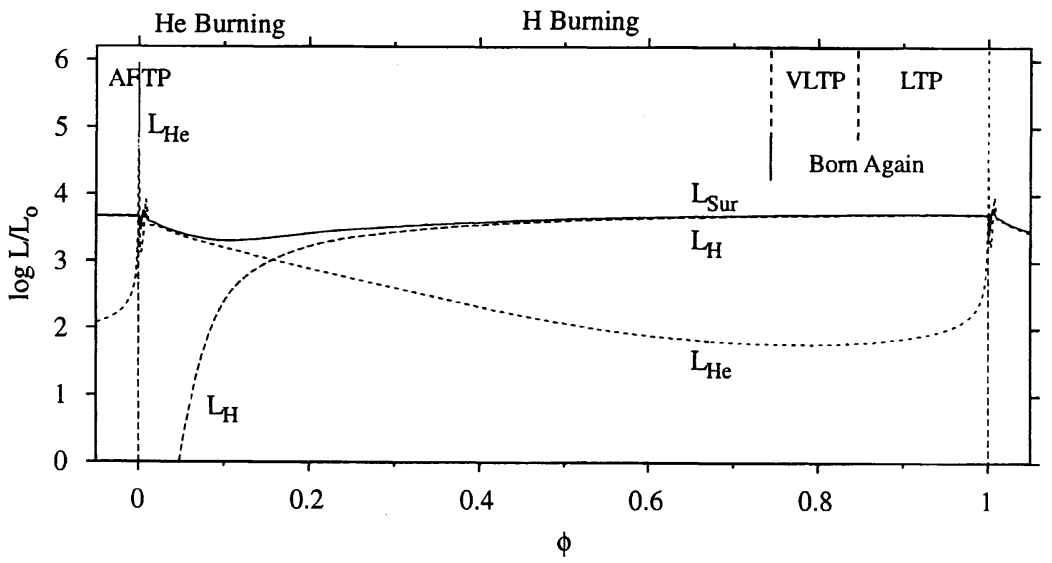

Figure 2. Luminosity contributions due to $\mathrm{H}\left(L_{\mathrm{H}}\right.$, dashed) and He burning ( $L_{\mathrm{He}}$, dotted) as well as surface lumininosity (solid) vs. thermal-pulse phase $\phi$ for the tenth pulse cycle of a $3 \mathrm{M}_{\odot}$ AGB sequence (Blöcker 1995a). Critical phase ranges for the occurence of thermal pulses during (AFTP) and after (LTP, VLTP) moving off the AGB are indicated (see text). 
transition times ( $\left.>10^{4} \mathrm{yr}\right)$ which increase with remnant mass. Blöcker (1995b) kept the high AGB rate until the star's fundamental radial pulsation period has fallen below 50 days (including a short reduction phase; $T_{\text {eff }}>5000 \mathrm{~K}$ ). Accordingly, mass loss shuts down the later the more massive the remnants are, leading to a very rapid decrease of the transition times $\left(<10^{4} \mathrm{yr}\right)$ with remnant mass. The transition times should not be too long since the coolest post-AGB stars known have effective temperatures of about $5000 \mathrm{~K}$ (Schönberner 1997), and kinematical ages of the youngest planetaries are only of the order of 1000 years. Fig. 1 shows the envelope mass for different remnant masses as a function of the effective temperature.

\section{From central stars to white dwarfs}

For the evolution through the PN region, mass loss can be described by the radiation-driven wind theory (Pauldrach et al. 1988). An adaption yields $\dot{M}_{\mathrm{CPN}}=1.3 \cdot 10^{-15} L^{1.9}$ (Blöcker $1995 \mathrm{~b}$ ), leading to rates of $10^{-8}$ to $10^{-7} \mathrm{M}_{\odot} / \mathrm{yr}$ for remnants of 0.6 to $0.8 \mathrm{M}_{\odot}$ being only of importance for massive remnants. Since $\Delta M_{\mathrm{e}}$ decreases and $\dot{M}_{H}$ increases with increasing core mass (see Fig. 1), more massive H-burning remnants evolve faster than less massive ones along the horizontal part of the evolution. Typical crossing times to evolve from $10^{4} \mathrm{~K}$ to maximum effective temperature amount to $\sim 10^{5} \mathrm{yr}$ for $0.55 \mathrm{M}_{\odot}, 4000 \mathrm{yr}$ for $0.6 \mathrm{M}_{\odot}, 800 \mathrm{yr}$ for $0.7 \mathrm{M}_{\odot}, 350 \mathrm{yr}$ for $0.84 \mathrm{M}_{\odot}$, and $50 \mathrm{yr}$ for $0.94 \mathrm{M}_{\odot}$. The crossing timescale of He burning central stars formed in the born-again scenario is roughly three times larger.

The "plateau" evolution at almost constant luminosity is terminated when the envelope mass becomes too small for hydrogen burning to be maintained. Then, the surface luminosity rapidly declines by more than one order of magnitude until it can be covered by gravothermal energy releases. He burning ceases as well in the course of evolution for most thermal-pulse phases. The further fading along the white dwarf cooling branch is controlled by gravothermal energy releases and neutrino losses and depends on the remnants' thermomechanical structure Though the evolutionary lines of degenerate cores belonging to different initial masses do, in principle, converge in the density-temperature plane (Paczynksi 1970), implying that the fading into white dwarfs depends only on the core mass, mass-loss terminates the AGB evolution well before such a convergence is reached (see Blöcker 1995a). Consequently, the thermomechanical structure and thus the fading times depend both on the core mass and the intial mass and thus on the complete evolutionary history. Only after sufficient cooling (e.g. $10^{6} \mathrm{yr}$ ), long after dispersion of the planetary nebula, the evolution becomes more and more independent of the remnant's history.

The detailed fading into a white dwarf is intimately linked to the degeneracy of the core. During the AGB evolution the degeneracy of the core increases and an increasing fraction of the energy which is released by contraction is used up by raising the Fermi energy of the electrons, being no longer available for the increase of the thermal energy of the star. Thus, the more degenerate a model leaves the AGB, i.e. the more compact and cooler the interior, the faster it fades after exhaustion of nuclear burning. For instance, since for a given initial mass the mean degeneracy increases with increasing AGB duration, models based on 


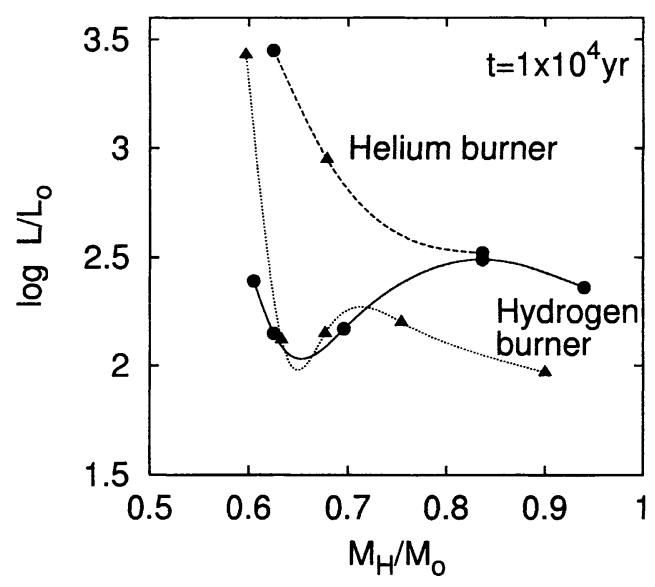

Figure 3. Luminosity vs. core mass at $t=10^{4} \mathrm{yr}$ for $\mathrm{H}$ burning (solid and dotted line) and He-burning models (dashed line) of Vassiliadis \& Wood (1994) [triangles] and Blöcker (1995b) [circles].

only one single initial mass (Paczynski 1971, Wood \& Faulkner 1986) fade the faster the more massive they are. However, considering reasonable initial-final mass combinations, more massive remnants can fade slower than less massive ones (Blöcker \& Schönberner 1990, Blöcker 1995b) since their progenitors have a less compact and hotter interior than those of lighter ones. The dependence of the fading times on the (poorly known) AGB mass-loss history is underlined by a comparison with the models of Vassiliadis \& Wood (1994), which are also consistent with initial-final mass relations. Their models dim faster than those of Blöcker (1995b), because they are based on AGB models which spent a longer time on the AGB due to smaller mass-loss rates and are thus more degenerate.

Fig. 3 shows the luminosity vs. remnant mass for both evolutionary sets at a post-AGB age of $t=10^{4} \mathrm{yr}$, the typical PN lifetime, and illustrates that the least luminous central stars should have masses of $\sim 0.65 \mathrm{M}_{\odot}$ (Blöcker \& Schönberner 1990). The position of He-burning models is also shown in Fig. 3. For lower masses, they evolve much slower than H-burning models. For larger remnant masses, it is hard to distinguish between the He-burning and the slowly fading H-burning model. Thus, in the Vassiliadis \& Wood scenario massive and luminous central stars are most likely He-burning objects whereas the calculations of Blöcker (1995b) suggest that they can also be explained by H-burning remnants.

\section{Hydrogen-deficient central stars}

The origin and evolution of H-deficient post-AGB star appeared to be enigmatic for many years. Although stars evolving through the AGB phase stay H-rich at their surfaces, a considerable fraction of their descendants show $\mathrm{H}$-deficient compositions (Mendez 1991). Approximately $20 \%$ of the whole CSPNe population seem to be $\mathrm{H}$-deficient while the rest show solar-like compositions. Important constituents of the H-deficient population are the Wolf-Rayet (WR) central stars and the hot PG 1159 stars with typical surface abundances of $(\mathrm{He}, \mathrm{C}, \mathrm{O})=(33,50,17)$ by mass (Dreizler \& Heber 1998, Koesterke \& Hamann 1997). Standard stellar evolution calculations failed to model these objects since they predict post-AGB stars either to have H-rich surfaces (e.g. Blöcker \& Schönberner 1997) or, if H-deficient, to expose only a few percent of oxygen in 


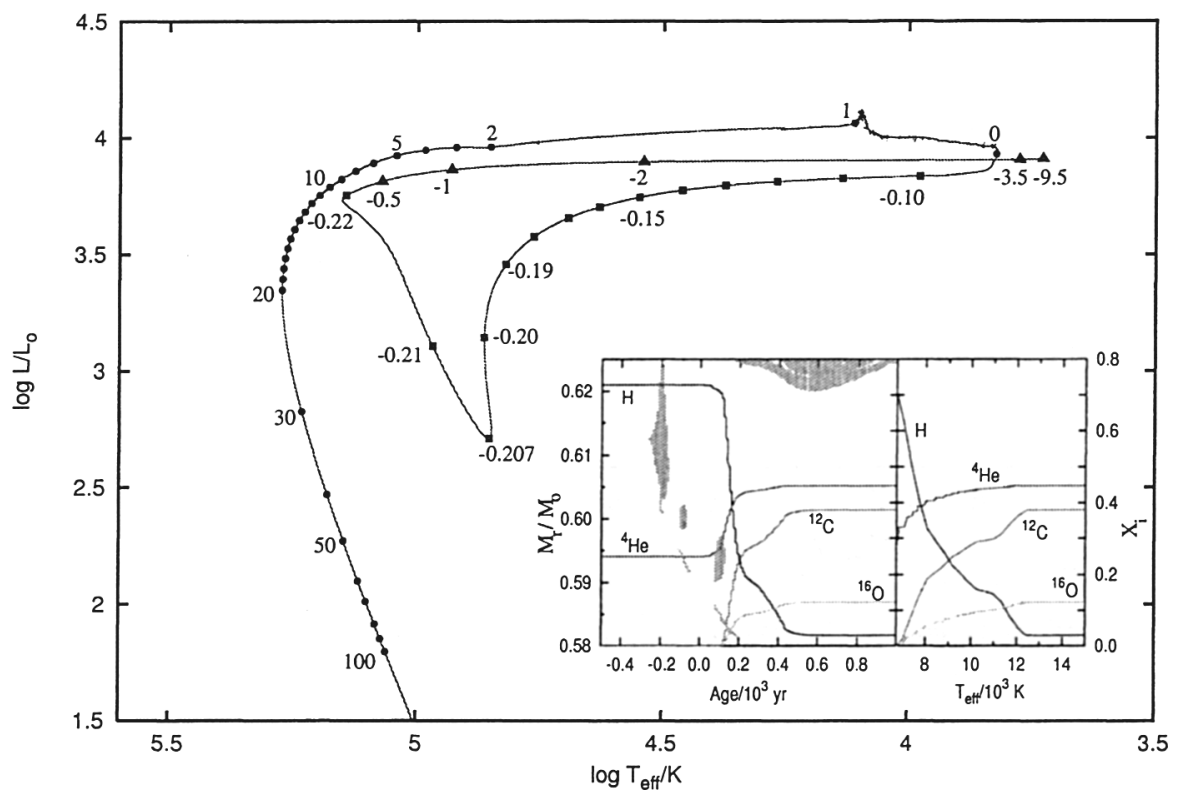

Figure 4. Evolutionary track for a $0.625 \mathrm{M}_{\odot}$ overshoot model suffering from an LTP (Blöcker 2001). Time marks are given in units of $10^{3} \mathrm{yr}$. Time is set to zero at minimum effective temperature after the flash. The age $-3500 \mathrm{yr}$ marks the begin of the central-star evolution and refers to a pulsational period of $50 \mathrm{~d}$. The inlet shows: Left: Extension of convective regions (left scale, shaded) and surface abundances of $\mathrm{H}, \mathrm{He}, \mathrm{C}$, and $\mathrm{O}$ (right scale) vs. time during flash and dredge up. Right: The surface abundances vs. $T_{\text {eff }}$ for the dredge-up phase.

their photospheres (Iben \& McDonald 1995). However, if convective overshoot is considered, H-deficient post-AGB stars with abundance patterns as observed in WR central stars can be formed (Herwig et al. 1999, Blöcker 2001, Herwig 2001a).

Herwig et al. (1997) showed that diffusive overshoot (Freytag et al. 1996) applied to all convective regions leads for AGB models to (i) efficient dredgeup and formation of low-mass carbon stars; (ii) formation of ${ }^{13} \mathrm{C}$ as a neutron source to drive $s$-process nucleosynthesis; and (iii) considerable changes of the intershell abundances The latter finding (iii) turned out to be a key ingredient for the modelling of WR central stars. Overshoot leads to an enlargement of the pulse-driven convection zone and to enhanced mixing of core material from deep layers below the He shell to the intershell zone ("intershell dredge-up") resulting in intershell abundances (mass fractions) of $(\mathrm{He}, \mathrm{C}, \mathrm{O})=(40,40,16)$ instead of $(70,25,2)$ as in non-overshoot sequences. These modified intershell abundances are already close to the observed surface abundances of Wolf-Rayet central stars. Finally, in contrast to standard evolutionary calculations, overshoot models do show dredge up for very low envelope masses, and efficient dredge up was found even during the post-AGB stage (Blöcker 2001, Herwig 2001a) leading to the mixing of the intershell abundances to the surface and to the dilution of hydrogen. Three thermal pulse scenarios for Wolf-Rayet central stars can now be 
distinguished:

AGB Final Thermal Pulse (AFTP), occurring immediately before the star moves off the AGB In this case the envelope mass is already very small $\left(\sim 10^{-2} \mathrm{M}_{\odot}\right.$, Fig. 1). During dredge-up a substantial fraction of the intershell region is mixed with the tiny envelope leading to the dilution of hydrogen and enrichment with carbon and oxygen. The resulting surface abundances depend on the actual envelope mass at which the AFTP occurs. For instance, Herwig (2001a) found for $\mathrm{M}_{\text {env }}=4 \cdot 10^{-3} \mathrm{M}_{\odot}(\mathrm{H}, \mathrm{He}, \mathrm{C}, \mathrm{O})=(17,33,32,15)$ after an AFTP. To obtain a sufficiently high probability for the AFTP $(\phi \approx 0$, Fig. 2$)$ requires, however, a coupling of mass loss to the thermal pulse cycle. The AFTP leads to a relatively high hydrogen abundance $(\gtrsim 15 \%)$ and predicts small kinematical ages for the PNe of WR central stars which emerge here directly from the AGB. Late Thermal Pulse (LTP), occurring when the model evolves with roughly constant luminosity from the AGB towards the white dwarf domain $(\phi \gtrsim 0.85$, Fig. 2). This kind of thermal pulse is similar to an AFTP but the envelope mass is even smaller $\left(\sim 10^{-4} \mathrm{M}_{\odot}\right.$, Fig. 1$)$. Fig. 4 shows the evolutionary track of a $0.625 \mathrm{M}_{\odot}$ LTP model with diffusive overshoot (Blöcker 2001). After the flash the intershell abundances amount to $(\mathrm{He}, \mathrm{C}, \mathrm{O})=(45,40,13)$ and the model evolves towards the AGB domain on a timescale of $\sim 100 \mathrm{yr}$. At minimum effective temperature $(\approx 6700 \mathrm{~K})$ dredge up sets in and continues until the star has reheated to $\approx 12000 \mathrm{~K}$. Hydrogen is diluted to $3 \%$ and the final surface abundances of $\mathrm{He}, \mathrm{C}$ and $\mathrm{O}$ are close to those of the intershell region, viz. $(45,38,12)$. Extension of convective regions and abundances are illustrated in the inlet of Fig. 4 as function of age and effective temperature, resp. The kinematical age of the PN amounts to a few thousand years. An observed example of an LTP is the born-again object FG Sge (Blöcker \& Schönberner 1997, Blöcker 2001).

Very Late Thermal Pulse (VLTP), occurring when the model is already on the white dwarf cooling track, i.e. after the cessation of $\mathrm{H}$ burning $(0.75 \lesssim \phi \lesssim$ 0.85 , Fig. 2). Then, the pulse-driven convection zone can reach and penetrate the H-rich envelope and protons are ingested into the hot, carbon-rich intershell region raising a H flash (Fujimoto 1977, Schönberner 1979, Iben \& McDonald 1995). The energy released by this flash leads to a splitting of the convection into an upper zone powered by $\mathrm{H}$ burning and a lower one powered by He burning. The upper convection zone is, however, short-lived because the available hydrogen in the envelope is quickly consumed. Finally, the star becomes hydrogen-free and exposes its intershell abundances at the surface. Herwig et al. (1999) found for a $0.604 \mathrm{M}_{\odot}$ overshoot model surface abundances of $(\mathrm{He}, \mathrm{C}, \mathrm{O})=(38,36,17)$. The kinematical age of the PN is relatively high since the star has first to fade along the cooling branch down to a few $100 \mathrm{~L}_{\odot}$ before the flash sets in. For 0.6 $\mathrm{M}_{\odot}$ one obtains typically $t \gtrsim 20000 \mathrm{yr}$. The central star V 4334 Sgr is thought to have recently suffered from a VLTP (Herwig 2001b).

All scenarios lead to hydrogen-deficient post-AGB stars with carbon and oxygen abundances as observed for Wolf-Rayet central stars. The variety of observations requires most likely all of these scenarios. Many objects have only very low hydrogen abundances, if any, favoring the LTP and VLTP. On the other hand, several Wolf-Rayet central stars are surrounded by young planetary nebulae (Tylenda 1996) and circumstellar shells consisting of both C- and O-rich material (Waters et al. 1998, Cohen et al. 1999) strengthening the AFTP and 
LTP. Within the current models roughly 20 to $25 \%$ of the stars moving off the AGB can be expected to become hydrogen-deficient.

\section{References}

Blöcker T., 1995a, A\&A 297, 727

Blöcker T., 1995b, A\&A 299, 755

Blöcker T., 2001, Ap\&SS 275, 1

Blöcker T., Schönberner D., 1990, A\&A 240, L11

Blöcker T., Schönberner D., 1997, A\&A 324, 991

Cohen M., Barlow M.J., Sylvester R.J., Liu X.-W., Cox P., Lim T., Schmitt B., Speck A.K., 1999, ApJ 513, L135

Dreizler S. \& Heber U., 1998, A\&A 334, 618.

Freytag B., Ludwig H.-G., Steffen M. (1996), A\&A 313, 497

Fujimoto M.Y., 1977, PASJ 29, 331

Habing H.J., 1996, A\&AR 7, 97

Herwig F., 2001a, Ap\&SS 275, 15

Herwig F., 2001b, ApJ 554, L71

Herwig F., Blöcker T., Schönberner D., El Eid, M., 1997, A\&A 324, L81

Herwig F., Blöcker T., Langer N., Driebe T., 1999, A\&A 349, L5

Iben I. Jr., 1984, ApJ 277, 333

Iben I. Jr., 1995, Physics Reports 250, 1

Iben I. Jr. \& MacDonald J., 1995, White dwarfs, Lecture Notes in Physics 443, D. Koester, K. Werner (eds.), Springer, p. 48

Koesterke L. \& Hamann W.R., 1997, A\&A 320, 91

Mendez R.H, 1991, Evolution of Stars: The Photospheric Abundance Connection, IAU Symp. 145, G. Michaud, A. Tutokov (eds.), Kluwer, p. 375

Paczyński B., 1970, Acta Astron. 20, 47

Paczyński B., 1971, Acta Astron. 21, 417

Pauldrach A., Puls J., Kudritzki R.P., Méndez R., Heap S.R.: 1988, A\&A 207, 123

Perinotto, M.: 1989, Planetary Nebulae, IAU Symp. 131, S. Torres-Peimbert, Ed., Kluwer, p. 293

Schönberner D., 1979, A\&A 79, 108

Schönberner D., 1983, ApJ 272, 708

Schönberner D., 1997, Planetary Nebulae, IAU Symp. 180, H.J. Habing \& H.J.G.L.M. Lamers (eds.), Kluwer, p. 379

Schwarzschild M. \& Härm, R., 1965, ApJ 142855.

Tylenda R, 1996, H-deficient stars, C.S. Jefferey \& U. Heber (eds.), ASP Conf. Ser. 96, 267

Vassiliadis E. \& Wood, P.R., 1993, ApJ 413, 641

Vassiliadis E. \& Wood, P.R., 1994, ApJS 92, 125

Waters L.B.F.M, Beintema D.A., Zijlstra A.A., de Koter A., Molster F.J., Bouwman J., de Jong T., Pottasch S.R., de Graauw T., 1998, A\&A 331, L61.

Weigert A., 1966, Z. Astrophys. 64, 395

Wood, P.R., 1997, Planetary Nebulae, IAU Symp. 180, H.J. Habing \& H.J.G.L.M. Lamers (eds.), Kluwer, p. 297

Wood P.R. \& Faulkner D.J., 1986, ApJ 307, 659 